\title{
IX. On algebraical couples
}

\section{Arthur Cayley Esq. B.A. F.C.P.S.}

To cite this article: Arthur Cayley Esq. B.A. F.C.P.S. (1845) IX. On algebraical couples, Philosophical Magazine Series 3, 27:177, 38-40, DOI: 10.1080/14786444508645221

To link to this article: http://dx.doi.org/10.1080/14786444508645221

册 Published online: 30 Apr 2009.

Submit your article to this journal 준

Џll Article views: 2

Q View related articles $₫$ 
IX. On Algebraical Couples. By Arthur Cayley, Esq., B.A., F.C.P.S., Fellow of Trinity College, Cambridge*.

$\mathrm{T} T$ is worth while, in connection with the theory of quater1 nions and the researches of Mr. Graves (Phil. Mag., No. 173), to investigate the properties of a couple $1 x+j y$, in which $i, j$ are symbols such that

$$
\begin{aligned}
& \imath^{2}=\alpha_{\imath}+6 j \\
& i j=\alpha^{1} \imath+b^{1} j \\
& j_{\imath}=\gamma_{\imath}+\delta j \\
& j^{2}=\gamma^{1} \imath+\delta^{1} j \text {. } \\
& \text { If } \overline{\imath x+j y} \bar{\imath}_{x_{1}+j y_{1}}=\imath \mathrm{X}+j \mathrm{Y} \text {, then } \\
& \mathrm{X}=\alpha x x_{1}+\alpha^{\mathrm{j}} x y_{1}+\gamma x_{1} y+\gamma^{1} y y_{1} \text {, } \\
& \mathrm{Y}=b x x_{1}+b^{1} x y_{1}+\delta x_{1} y+\delta^{1} y_{1} \text {. }
\end{aligned}
$$

Imagine the constants $\alpha, b \ldots$ so determined that,$x+j y$ may have a modulus of the form $\mathrm{K}(x+\lambda y)(x+\mu y)$; there results one of the four following essentially independent systems:-

A.

$$
\begin{aligned}
& \iota^{2}=\frac{1}{\lambda \mu}(\delta \lambda \mu+\gamma \overline{\lambda+\mu}) \iota-\frac{\gamma}{\lambda \mu} j \\
& { }_{\imath} j=j \imath=\gamma \imath+\delta j \\
& j^{2}=-\lambda \mu \delta i+(\gamma+\overline{\lambda+\mu} \delta) j \\
& \left\{\begin{array}{l}
\mathrm{X}+\lambda \mathrm{Y}=\frac{1}{\lambda}(\gamma+\lambda \delta)(x+\lambda y)\left(x_{1}+\lambda y_{1}\right) \\
\mathrm{X}+\mu \mathrm{Y}=\frac{1}{\mu}(\gamma+\mu \delta)(x+\mu y)\left(x+\mu y_{1}\right) .
\end{array}\right.
\end{aligned}
$$

The couple may be said to have the two linear moduli,

$$
\frac{1}{\lambda}(\gamma+\lambda \delta)(. x+\lambda y): \frac{1}{\mu}(\gamma+\mu \delta .)(x+\mu y) ;
$$

as well as the quadratic one,

$$
\frac{1}{\lambda \mu} \overline{\gamma+\lambda} \delta \overline{\gamma+\mu} \delta \overline{x+\lambda} y \overline{x+\mu} y,
$$

the product of these, which is the modulus, and the only modulus in the remaining systems.

B.

$$
\begin{aligned}
\iota^{2} & =-\delta \imath+\frac{1}{\lambda \mu}(\gamma+\delta \overline{\lambda+\mu}) j \\
\imath j & =j \imath=\gamma \iota+\delta j \\
j^{2} & =(\overline{\lambda+\mu} \gamma+\lambda \mu . \delta) \iota-\gamma j \\
& * \text { Communicated by the Author. }
\end{aligned}
$$


Mr. A. Cayley on Algebraical Couples.

$$
\left\{\begin{array}{l}
\mathrm{X}+\lambda \mathrm{Y}=\frac{1}{\mu}(\gamma+\lambda \delta)(x+\mu y)\left(x_{1}+\mu y_{1}\right) \\
\mathrm{X}+\mu \mathrm{Y}=\frac{1}{\lambda}(\gamma+\mu \delta)(x+\lambda y)\left(x_{1}+\lambda y_{1}\right) .
\end{array}\right.
$$

C. $\iota^{2}=\frac{1}{\lambda \mu}(\delta \lambda \mu+\gamma(\overline{\lambda+\mu})) \bullet-\frac{\gamma}{\lambda \mu} j$

$$
\begin{aligned}
& \qquad j=\left(\frac{\mu^{2}+\mu \lambda+\lambda^{2}}{\mu \lambda} \gamma+\overline{\mu+\lambda \delta}\right) \cdot+\left(-\delta-\frac{\lambda+\mu}{\lambda \mu} \gamma\right) j \\
& j ı=\gamma ı+\delta j \\
& j^{2}=(\overline{\lambda+\mu} \gamma+\lambda \mu \delta) \iota-\gamma \jmath \\
& \left\{\begin{array}{l}
\mathrm{X}+\lambda \mathrm{Y}=\frac{1}{\lambda}(\gamma+\lambda \delta)(x+\lambda y)\left(x_{1}+\mu y_{1}\right) \\
\mathrm{X}+\mu \mathrm{Y}=\frac{1}{\mu}(\gamma+\mu \delta)(x+\mu y)\left(x_{1}+\lambda y_{1}\right) .
\end{array}\right.
\end{aligned}
$$

D. $\iota^{2}=-\delta i+\frac{1}{\lambda \mu}(\gamma+\delta \overline{\lambda+\mu}) j$

$$
\begin{aligned}
& \qquad j=(-\gamma-\overline{\lambda+\mu} \delta) \iota+\left(\frac{\lambda+\mu}{\lambda \mu} \gamma+\frac{\lambda^{2}+\lambda \mu+\mu^{2}}{\lambda \mu} \delta\right) \jmath \\
& j ı=\gamma \iota+\delta j \\
& j^{2}=-\lambda \mu \delta i+(\gamma+\overline{\lambda+\mu} \delta) j \\
& \left\{\begin{array}{l}
\mathrm{X}+\lambda \mathrm{Y}=\frac{1}{\mu}(\gamma+\lambda \delta)(x+\mu y)\left(x_{1}+\lambda y_{1}\right) \\
\mathrm{X}+\mu \mathrm{Y}=\frac{1}{\lambda}(\gamma+\mu \delta)(x+\lambda y)\left(x_{1}+\mu y_{1}\right) .
\end{array}\right.
\end{aligned}
$$

The formulæ are much simpler and not essentially less general, if $\mu=-\lambda$. They thus become

$\mathbf{A}^{\prime}$.

$$
\begin{aligned}
\imath^{2} & =\delta \imath+\frac{\gamma}{\lambda^{2}} j \\
\imath & =j \imath=\gamma \iota+\delta j \\
\dot{j}^{2} & =\lambda^{2} \delta i+\gamma j \\
\mathrm{X} \pm \lambda \mathrm{Y} & = \pm \frac{1}{\lambda}(\gamma \pm \lambda \delta) \cdot(x \pm \lambda y)\left(x_{1} \pm \lambda y_{1}\right) .
\end{aligned}
$$

(Two linear moduli.)

B'.

$$
\begin{aligned}
\iota^{2} & =-\delta \imath-\frac{\gamma}{\lambda^{2}} j \\
\iota & =j \iota=\gamma ı+\delta j \\
j^{2} & =-\lambda^{2} \delta_{\imath}-\gamma j \\
\mathrm{X} \pm \lambda \mathrm{Y} & =\mp \frac{1}{\lambda}(\gamma \pm \lambda \delta)(x \mp \lambda y)\left(x_{1} \mp \lambda y_{1}\right) .
\end{aligned}
$$


C.

$$
\begin{aligned}
& \imath^{2}=-\delta \imath+\frac{\gamma}{\lambda^{2}} j \\
& \jmath=-\gamma \iota-\delta j \\
& j \iota=\gamma_{\imath}+\delta j \\
& j^{2}=-\lambda^{2} \delta_{l}-\gamma j \\
& \mathrm{X} \pm \lambda \mathrm{Y}= \pm \frac{1}{\lambda}(\gamma \pm \lambda \delta)(x \pm \lambda y)\left(x_{1} \mp \lambda y_{1}\right) . \\
& \imath^{2}=-\hat{\delta} \imath-\frac{\gamma}{\lambda^{2}} j \\
& j=-\gamma \iota-\delta j \\
& j_{i}=\gamma i+\delta j \\
& j^{2}=\lambda^{2} \delta_{\imath}+\gamma j \\
& \mathrm{X} \pm \lambda \mathrm{Y}=\mp \frac{1}{\lambda}(\gamma \pm \lambda \delta .)(x \mp \lambda y)\left(x_{1} \pm \lambda y\right) \text {. }
\end{aligned}
$$

D'.

There is a system more general than (A.) having a single linear modulus $q(\theta \mathrm{X}+\mathrm{Y})$ : this is E.

$$
\begin{aligned}
\imath^{2} & =a(\iota-\theta j)+\theta^{2} q j \\
\imath j & =\alpha^{\prime}(\iota-\theta j)+\theta q j \\
j \imath & =\gamma(\iota-\theta j)+\theta q j \\
j^{2} & =\gamma^{\prime}(\iota-\theta j)+q j \\
\theta \mathrm{X}+\mathrm{Y} & =q(\theta x+y)\left(\theta x_{1}+y_{1}\right) ;
\end{aligned}
$$

or, without real loss of generality,

E'.

$$
\begin{gathered}
\iota^{2}=\alpha i \\
\imath j=\alpha^{\prime} \\
j \iota=\gamma^{\prime} \\
j^{2}=\gamma^{\prime}+q j . \\
\mathrm{Y}=q y y_{1} .
\end{gathered}
$$

To complete the theory of this system, one may add the identical equation

$$
\begin{aligned}
& \mathrm{X}+\frac{1}{\theta-q \mathrm{M}} \mathrm{Y}=\frac{q^{\left(\theta^{2}-\mathrm{M} \alpha\right)}}{\theta-q \mathrm{M}}\left(x+\frac{\alpha^{\prime}-\theta \gamma}{\alpha-\theta \alpha^{\prime}} y\right)\left(x_{1}+\frac{\alpha^{\prime}-\theta \gamma^{\prime}}{\alpha-\theta \gamma} y_{1}\right), \\
& \text { where } \quad \mathrm{M}=\frac{\theta\left(\gamma-\theta \gamma^{\prime}\right)-\left(\alpha-\theta \alpha^{\prime}\right)}{\alpha^{\prime} \gamma-\alpha \gamma^{\prime}} .
\end{aligned}
$$

By determining the constants, so that $\frac{1}{\theta-q M}=\frac{\alpha^{\prime}-\theta \gamma}{\alpha-\theta \alpha^{\prime}}$ $=\frac{a^{\prime}-\theta \gamma^{\prime}}{\alpha-\theta \gamma}$, the system would reduce itself to the form $A$.

Cam bridge, April 23, 1845. 